- Aumentar la oportunidad de que los alumnos puedan realizar inmediatamente experiencias cientlficas gratificantes.

El project Physics se compone de un libro de texto (textbook), un libro del Profesor (Resource Book), un libro de experiencias (Handbook) y una serie de Lecturas (Readings) y de material audiovisual auxiliar. Ha sido traducido a numerosos idiomas (frances, por. itugués, italiano, etc.) sin que por el momento exista traducción en ninguna de las lenguas del Estado Espantol. Proyectos en torno al «Project
Physics»

Los objetivos del Project Physics, en la línea de dar una visión humanista de la ciencia y situar al alumno en el proceso histórico en el que han tenido lugar los descubrimientos científicos, coinciden en gran parte con los que animan a muchos profesores de física $y$ grupos de didáctica que están trabajando en nuestro pais. No es, pues, de extrantar que a raiz de las conferencias dadas por el profesor G. Holton en Madrid y Barcelona durante el curso pasado, surgiera, en la Comisión de Física y Quimica del Colegio de Licenciados de Cataluña la idea de realizar la traducción y adaptación de este proyecto.

En la actualidad et Grupo RecercoFaraday del 1.C.E. de la Universidad Autónoma de Barcelona está elaboran. do una serie de programas-guía (serie de actividades secuenciadas, que engloba cuestiones, lecturas, experimentos, problemas, etc.) de cada uno de los ca. pítulos del texto que corresponden al programa de física de $2^{\circ}$ de BUP: E] lenguaje del movimiento, Caida libre: Galileo describe el movimiento, El nacimiento de la dinámica, Energia y electromagnetismo. Estos programas-gufa se están experimentando en el presente curso, con el fin de realizar una valoración crítica de cada unidad, que permita conocer las ventajas y posibles dificultades que pueda presentar la adaptación del Project Physics en nuestro bachillerato.

Otro grupo del I.C.E. de la U.A. de Barcelona, el Taller de experiencias de Hostafranchs se ha comprometido a realizar y adaptar en el presente curso los experimentos del Handbook def Project Physics, correspondientes a los temas de mecánica y electricidad.

Por último el grupo de Historia de la Física y la Química del Colegio de $\mathrm{Lj}$ cenciados de Cataluña ha venido manteniendo contacto con el profesor G. Holton con objeto de traducir y adaptar el Project Physics al catalán. Para ello se piensa solicitar una subvención de la Universidad Autonoma de Bar. ceiona. En una segunda fase podría traducirse al castetiano, si se encontrara una editorial dispuesta a realizar la edición.

Confiemos pues, que podamos contar en oreve tiempo con la adaptación del Project Physics en catalán y casteliano, y que pueda realizarse una adecuada difusión eatre el profesorado de ciencias de BUP, COU, FP y Escuelas Normales y una experimentación en los niveles correspondientes.

AURELI CAAMAÑo (De la Comisión de Física y Quimica del Colegio de Licenciados de Cataluña)

\section{RESEARCH, ANYONE?}

Robert BAUMAN, 1983. The Physics Teacher, vol. $21 \mathrm{pp} .77$ y 113 .

Se trata de un editorial firmado por el Presidente de la American Association of Physics Teacher destinado a analizar la investigación didáctica que viene realizándose en el campo de la Física.

Comienza señalando que la mayor parte de los trabajos que se presentan como investigación educativa son únicamente estudios descriptivos que, aunque pueden tener interts, no son verdaderas investigaciones.

Pone después de relieve que los mayores esfuerzos cn investigación educativa han esiado centrados en los méto. dos de ensenanza, mientras que se ha investigado poco, hasta recientemente, en los procesos de aprendizaje. Y es aqui, en opinion de Bauman, donde se necesita más investigación. Sugiere a cs. te respecto partir del modelo de desa. trollo mental elaborado por Piaget que "permite comprender por qué y en qué dominios los estudiantes tienen dificultades y proporciona pruebas con las que determinar en qué medida hemos ayudado a los alumnos a desarrollarse. Nos queda ahora encontrar las tec. nicas que puedan favorecer el necesario desarrollo mental abriendo las vias para que ruestros alumnos compren. dan los conceptos físicos." Y termina:

«Este es un programa de investigación que merece los esfuerzos de cualquier profesorn.

D.G.

\title{
PUBLICACIONES RECIBIDAS
}

PUBLICACIONES DEL I.C.E. DE LA UNIVERSIDAD DE CORDOBA

- Curso de Prácticas de Física en B.U.P. y C.O.U.:

1. La camara fotografica y el estrobos. copio. Aplicaciones prácticas.

2. Banco de aire. Aplicaciones prácricas.

Autores: E. Castaflos Soler F. Garcla Jimenez E. González Román J. Martinez Aguilar
- Itinerarios Geológicos de la Provincia de Córdoba como ejemplo del interés de las actividades de campo en la Enseñanza de la Geologia (Nivel Bachillerato y C.O.U.)

Autores: Grupo de Trabajo del Seminario Permanente de Ciencias Naturales.

\section{PUBLICACIONES DEL} I.C.E. DE LA UNIVERSIDAD DE EXTREMADURA

- El Análisis Matemático en B.U.P. y C.O.U.

Autores: C. Benitez Rodriguez E. López Ayuso

PUBLICACIONES DEL MINISTERIO DE EDUCACION Y CIENCIA

Etias Fernández Uria, 1979. Estructuro y didáctica de las Ciencias.

Tomás Escudero Escorza, 1979. Ensehanza de la Fisica en la Universidad. 
Varios Autores, 1982. Lógica, epistemologia y teoría de la ciencia.

Maria L. Fernández Castañón y otros. PEAC (Proyecto Experimental Area Ciencias de la Naturaleza.

\section{Introducción (1980)}

2. Unidad O. Ténicas de observación $y$ medida (1980)

3. Monografia: La enseñanza por el entorno ambiental (1981)
4. Nicleo 1. Las fuerzas en la naturaleza (1981)

J. Garcia Rua y J.M. Martínez Sánchez, 1977. Matemática Básica Elemental.

Varios Autores, 1975. Ornitologia y conservación de la naruraleza hoy.

Aquilino Duque, 1979. El mito de Donana.
Colección Estudio y Experiencias Educativas (Serie EGB)

La ensettanza de las ciencias y sus relociones interdisciplinarias en la segunda etapa de EGB. 1971.

Educación y medio ambiente. Actividades y experiencias. 1981.

Matemáticas. 1981.

Ciencias de la naturaleza (II). 1981.

\section{SELECCIONES BIBLIOGRAFICAS TEMATICAS}

\section{LOS TRABAJOS PRACTI. COS DE FISICA Y DE OUI- MICA: UNA SELECCION BIBLIOGRAFICA}

A continuación presentamos una seleccion de la bibliografia relativa a los Trabajos Prácticos publicada en estos últimos años en las siguientes revistas.

Education in Chemistry (Educ. Chem.) European Journal of Science Education (Eur. J. Sci. Educ.), Journal of Chemical Education (J. Chem. Educ.), Journal of Research in Science Teaching (J. Res. Sci. Teach), Physics Education (Phys. Educ.), Revista de Bachillerato (Rev. Bach.), Revue Française de Pedagogie (Rev, Fran. Ped.), Science Education (Sci. Educ.), Soviet Education (Soviet Educ.).

La selección se presenta cronológicamente y abarca desde 1970 a 1982, sin pretender por supuesto que sea exhaus. tiva. Los comentarios que acompantan están realizados de acuerdo con nuestra concepcion de los Trabajos Práct:. $\cos$ como pequetas investigaciones que permiten mediante un descubrimiento guiado mejorar la adquisición significativa de conocimientos, así como conseguir una familiarización con la metodologla cientffica.

Charen, G. 1970, Do laboratory Methods Stimulate Critical Thinking, Sci Educ. 54, pp 267-271.

Rechaza las prácticas receta por no permitir el desarrollo del pensamiento $\mathrm{cr}$ tico. Propone el enfrentarse con problemas reales que necesiten ser atacados en el laboratorio y finalmente re- quieran de un informe escrito similar a los preparados en las investigaciones cientfficas

Belousov, V.A., 1973, Techniques for conducting an experiment in physics, Soviet Educ. Nov-Dic 1973, pp 116-121.

Hace referencia al papel potencial del experimento f́́sico como método de comprensión y fuente de conocimientos, mientras que a menudo los profesores lo utilizan como simple ilustracion de su transmision oral de conocimientos perdiéndose o mal utilizándose todas sus posibilidades.

MacDuffie, D.E, 1973, Learning Though Practical Work. Educ. Chem, Mayo 1973.

Reconoce los inconvenientes de la receta tradicional y en su lugar propone unos trabajos prácticos que presentan una introducción con sus bases teóri. cas acompantadas de un breve diseño del trabajo, seguido de una colección de hechos relevantes ordenados en una secuencia lógica (detalies técnicos, posibles fuentes de dificultad o azar, indicaciones de efectos posibies, omisiones importantes, etc.). Es decir propone ampliar «la receta», justificándola $y$ dejando algunos puntos para que ios resuelva el alumno.

Swan, R.L. 1974, Practical Objetives a Review Educ. Chem, Sept. 1974

Este artículo analiza algunos de los estudios realizados por varios autores en antos recientes sobre los objetivos de la prácticas de laboratorio. Gunning, D.J. y Johnstone, A.Y., 1 pp 315.317 .
1976. Practical Work in the Scottish Ograde. Educ. Chem. Ene. 1976, pp 12-14.

Describe los resultados de una investigacion sobre el papel de los trabajos prácticos en un proyecto escocés de renovación del aSyllabus» de quimica. El cambio más importante de este proyec. to lo introducen los trabajos prácticos. Reconoce la importancia que tiene el que sean los alumnos quienes lleven a cabo las experiencias por ellos mismos.

Johnstone, A.H. y Wood, C.A. 1977. Practical Work in its own right. Educ. Chem. 1977 pp 11-12.

Los autores intentan confrmat que los trabajos prácticos no tienen por qué adaptarse al contenido de un programa, sino que pueden estar basados en ellos mismos. Asi preparan unos cuestionarios basados sobre los objetivos de los trabajos prácticos que utilizan como herramienta para evaluar su planteamiento.

Racheison, S. 1977. A question of balanc: $A$ wholistic view of scientific inquiry. Sci. Educ. vol.61, pp 109.117. Pone en evidencia el desacuerdo entre la ensehanza de las ciencias y la metodología cientifica. La ensenanza de las ciencias está poniendo mucho enfasis en que los estudiantes se equipen con los conocimientos ténicos para realizar los experimentos sin preocuparse de las actividades que conducen a formular hipótesis creativas.

Pickering, M. y Golstein, S.L., 1977 The Educational Efficiency of Laboratory Reports. J. Chem. Educ. vol. 54 